\title{
Multiple void formation in plasmas containing multispecies charged grains
}

\author{
Y. H. Liu, ${ }^{1}$ Z. Y. Chen, ${ }^{1, *}$ M. Y. Yu, ${ }^{2}$ and A. Bogaerts ${ }^{1}$ \\ ${ }^{1}$ Department of Chemistry, University of Antwerp, Universiteitsplein 1, B-2610 Antwerp, Belgium \\ ${ }^{2}$ Theoretische Physik I, Ruhr-Universität Bochum, D-44780 Bochum, Germany
}

(Received 8 July 2006; published 13 November 2006)

\begin{abstract}
Self-organized separation of charged-dust species in two-dimensional dusty plasmas is studied by means of molecular-dynamics simulation. The multispecies dust grains, interacting through a screened Coulomb potential with a long-range attractive component, are confined by an external quadratic potential and subjected to a radially outward ion drag force. It is found that, in general, the species are spatially separated by bandlike dust-free (or void) regions, and grains of the same species tend to populate a common shell. At large ion drag and/or large plasma screening, a central disklike void as well as concentric bandlike voids separating the different species appear. Because of the outward drag and the attractive component of the dust-dust interaction forces, highly asymmetrical states consisting of species-separated dust clumps can also exist despite the fact that all the forces are either radial or central.
\end{abstract}

DOI: 10.1103/PhysRevE.74.056401

PACS number(s): 52.27.Lw, 36.40.Wa, 61.43.Bn, 61.50.Ah

\section{INTRODUCTION}

The behavior of micro- or nanometer-sized grains in a dusty (or complex) plasma has been extensively studied in the past few years [1-5]. The grains usually acquire a negative charge because of the high mobility of the electrons. When the grains are confined in an external potential, they can self-organize into a lattice structure and form dust crystals [6]. At higher temperatures, melting of the structure occurs and the dusts become liquidlike [7]. In many situations, voids, or local dust-free regions, appear in the plasma [8-11]. Voids can occur as three-dimensional (3D) as well as two-dimensional (2D) structures [8-17]. Laboratory observations suggest that the voids, once formed, are fairly robust, and their structures are independent of how they are formed. In general, the void size increases with the rf power input and the neutral gas pressure in the plasma discharge. Several mechanisms for void formation have been proposed and investigated [10-23].

Besides the external forces, such as that of the confining electric field, other forces on the dust grains include thermophoretic, space-charge, drag of the ions that are streaming toward the boundary of the plasma, as well as other collisional forces. Several theories also invoked ionization or other effects for triggering void formation [18-21]. Dustcontaining plasma systems are often called complex plasmas since many closely related effects are simultaneously present and can affect each other. It is thus often difficult to pinpoint the main mechanism for any specific process [10-23].

Recently, Huang et al. [15] and Huang [16] observed central as well as concentric shell-like voids separating different dust regions during the dust formation stage in a discharge plasma. In this stage, the dust particles are still too small to be individually observable with the available equipment and their structure appears only as bright (due to light scattering by the fine dust particles) and dark regions. In the later stage

\footnotetext{
*Present address: Department of Chemistry, George Washington University, Washington, DC 20052, USA
}

of the evolution, when the grains become large and uniform, the shell-like voids disappear and the dust distribution becomes homogeneous. Liu et al. [24] attributed the void structures in the evolution stage to the existence and separation of different dust species that are evolving in the discharge. Using molecular dynamics (MD) simulation, they considered a plasma containing several species of dust grains interacting through a pure Coulomb potential, together with a shortrange attractive component to model the effect of the background plasma. It was found that when the ion drag force, arising from the ubiquitous streaming of plasma ions to the walls of the discharge [10-13], on the grains is included, 2D disk- and bandlike voids can occur and coexist.

In a quiescent thermal plasma, the charged grains are always Debye screened, so that their electrostatic potential has a range of the order of the Debye length, i.e., much shorter than that of the very long-range Coulomb potential. In a dusty discharge, the dusts can still be evolving in different stages that are separated by different chemical and physical time scales; it is not clear if and how a charged dust grain is shielded. To formulate a self-consistent theory is difficult because of the presence of highly different time scales and the complex nature of the problem. Several novel models, with various combinations of shielded and unshielded, repulsive as well as attractive, potentials, can be found in the literature [25-33]. The possibility of attractive interaction force between like-charged grains in plasmas is a topic of many recent investigations. However, since the force between two grains is a collective instead of direct effect involving not only the background plasma but also the nearby dust grains, the exact nature and source of the apparent or effective attractive force remains unclear. For example, Tsytovich et al. [26,31] and Tsytovich and Morfill [29] showed that the shielding and scattering of impacting plasma ions by neighboring grains can result in an attractive force between the latter. Chen et al. [32] showed that an effective attractive force can appear if one assumes that grains being considered have a limited sphere (determined by complete charge neutralization within it) of influence. Wang [33] found that if neighboring grains have the same surface potential, a shielded attractive part can appear in the interaction 
potential. However, it is difficult to isolate any of the proposed mechanism for verification, and the experimental evidences of grain attraction are indirect $[34,35]$. Accordingly, the Debye-screened repulsive Coulomb potential of the grain in a thermal plasma is usually used as for convenience.

Of special interest are 2D multispecies dust structures in a plasma because they are useful as models for $2 \mathrm{D}$ crystals and clusters. Although the different species can have different mass and charge, in order to remain 2D they should have the same charge-to-mass ratio so that the downward gravity and the upward discharge electric force on the dust grains can balance to form a horizontal dust layer $[4,5]$. It is, thus, of interest to investigate multispecies dust grains having the same charge-to-mass ratio. In Ref. [24], systems of charged grains interacting through a potential containing a long-range (Coulomb) repulsive and a short-range attractive component were studied. However, in some popular models, the attractive interactions are either unscreened or screened at distances much larger than the usual Coulomb repulsive interaction (see Ref. [26] for a more detailed discussion); it is therefore also of interest to study grains with short-range repulsive and long-range attractive interactions. In order to obtain a better understanding of how the Debye screening of the Coulomb potential affects the formation and structure of the voids in a multispecies dust system, in contrast to Ref. [24], we shall study in this paper systems of charged grains interacting through a potential containing a screened Coulomb (Yukawa) repulsive component and a long-range attractive component, the latter representing effects such as dust shielding and shadowing $[29,32,33]$. To model typical laboratory complex discharge plasmas, the dust grains can have different composition and are externally confined by a quadratic trap, as well as subject to a radially outward drag force exerted on them by the plasma ions streaming outward to the discharge wall $[4,5,19-22]$.

\section{MODEL SYSTEM}

We consider a 2D system of charged dust grains, confined in an external quadratic trap [3,36-40]. The grains are allowed to have different chemical and physical composition. The dust grains interact through a screened Coulomb potential and a long-range attractive potential [25-27]. A radially outward force (opposite to the confinement force) models the drag force exerted on a dust grain by the high-speed ions streaming toward the plasma-wall boundary $[11-13,24]$. In the dimensionless form, the Hamiltonian of the system is $H=K+U$, where $K$ is the kinetic energy and

$$
U=\sum_{i=1}^{N}\left(m_{i}^{(k)} r_{i}^{2}-A q_{i}^{(k) 2} r_{i}\right)+\sum_{i<j}^{N} \frac{q_{i}^{(k)} q_{j}^{(k)}}{r_{i j}}\left(e^{-\kappa r_{i j}}-\alpha\right),
$$

is the potential energy. In Eq. (1), the units for the length and energy are $r_{0}=\left(2 Q^{(1) 2} / M^{(1)} \varepsilon \omega_{0}^{2}\right)^{1 / 3}$ and $E_{0}=M^{(1)} \omega_{0}^{2} r_{0}^{2} / 2$, respectively, where $\omega_{0}$ is the trapping frequency, and $\varepsilon$ (constant) is the dielectric constant of the background plasma, $Q^{(1)}$ and $M^{(1)}$ are the charge and the effective mass of species 1 , respectively. Since the dust grains of the same species are indistinguishable, we can drop the grain indices. Here,
$N=\sum_{k=1}^{n} N^{(k)}$ is the total number of grains, where $n$ is the total species number in the system, $N^{(k)}$ is the number of the grain species $k, \mathbf{r}_{i}$ is the position of the grain $i(=1, \ldots, N)$, $r_{i j}=\left|\mathbf{r}_{i}-\mathbf{r}_{j}\right|$, and $q_{i}^{(k)}$ and $m_{i}^{(k)}$ are the dimensionless charge (normalized by $Q^{(1)}$ ) and mass (normalized by $M^{(1)}$ ) of the grain $i$ of species $k$. In Eq. (1), the term involving $A$ represents the ion drag force potential [11,12]. Furthermore, $\kappa=r_{0} / \lambda_{D}$ determines the strength of the screening effect of the background plasma on the grain, $\lambda_{D}$ is the Debye screening length, and $\alpha$ determines the strength of the intergrain attraction [31]. In Eq. (1), the first term is the confinement potential, the second is the potential associated with the drag force, and the third and the last are the repulsive and the attractive parts of the intergrain potential, respectively. In the minimum-energy state, we have $H \rightarrow U$.

\section{SIMULATION RESULTS}

MD simulation is used to follow the evolution of the system. Each run is started with a random phase-space distribution of the dust grains at a fairly high temperature $(T=0.05$ $\rightarrow 1.0$, where $T$ is normalized by $E_{0}$ ). The system is annealed slowly until a minimum-energy state with temperature $T \sim 0 \pm 10^{-6}$ is reached. The accuracy of the simulation was checked by comparing the results to the ground-state configurations of single-species clusters [25] obtained by Monte Carlo (MC) simulations. As discussed in Ref. [26], the value of the attraction coefficient $\alpha(\leq 1)$ is usually small but can be as large as 0.6 , depending on the property of the grain surface as well as the local plasma conditions. Since it is uncertain how significant the intergrain attraction is in the band-void experiments $[15,16]$, we shall use $\alpha=0.01$ [26] in most of our cases. The grains have different charge and mass, but the same charge-to-mass ratio $q^{(k)} / m^{(k)}=1$ $(k=1, \ldots, n)$. That is, the different species represent grains of different material and/or density. We also note that the charge ratio of two dust species is equal to their mass ratio.

In the following, we shall first study the minimum-energy structures of systems $(N=150)$ with two dust species by varying either the drag-force strength $A$ or the screening strength $\kappa$. We then consider a larger system $(N=300)$ with three species of grains at different $A$ and screening strength $\kappa$. Finally, we shall investigate highly asymmetrical dust structures.

\section{A. Systems with two species of grains}

\section{Effect of ion drag}

We first consider an $N=150$ dust system containing two species of grains with different charge and mass but the same charge-to-mass ratio. Figure 1 shows typical minimumenergy dust configurations for $\kappa=4$ and different drag strength $A$. The case $q^{(2)}=q^{(1)}=1.0$ and $m^{(2)}=m^{(1)}=1.0$, i.e., the two species are physically indistinguishable, is included (Figs. 1(a1)-1(a4), first column) for reference and comparing with existing MC-simulation results of a single-species system [25]. In Fig. 1, the columns show the effect of the drag strength $A$ and the rows show the effects of the grain charge $q^{(2)}$ and mass $m^{(2)}$. All the subfigures are of the same 


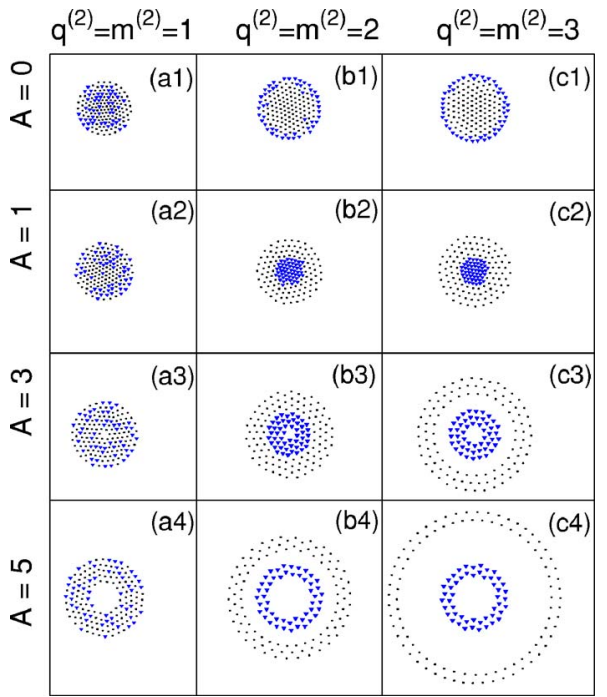

FIG. 1. (Color online) Minimum-energy configurations for twospecies of different charge and mass, but the same charge-to-mass ratio, for different drag force strength $A$ at a fixed screening strength $\kappa=4$. The total number of grains is $N=150$. The numbers of grains of species 1 (triangles) and 2 (dots) are $N^{(1)}=50$ and $N^{(2)}=100$, respectively. The system sizes in the subfigures are of the same arbitrary scale.

arbitrary scale. From Figs. 1(a1)-1(a4), one can see that when the two species are physically indistinguishable, only a central disklike void appears at the center. As expected, the sizes of the void and the overall structure increase with the (radially outward) drag force, or $A$.

From the second and third columns in Fig. 1, we can see that two physically distinct dust species tend to separate and occupy different regions in the plasma. When $A=0$, or no drag force, the heavier grains are accumulated at the system center because of the mass-dependent radially inward external confinement force. The details of the structure depend on the grain-grain interaction forces (here, $\alpha=0.01$ and $\kappa=4$ ) as well as energy minimization. When $A \neq 0$, grains of different species also occupy different radial regions, with the larger mass and charge located farther away from the center. This is because grains of larger charge and mass experience stronger outward drag force. The latter can exceed the inward confinement force, which is only mass dependent. From Fig. $1(\mathrm{~b} 4)$, one can see that for $q^{(2)}=2$ and $m^{(2)}=2$, a bandlike void region appears when $A=5$. From Fig. 1(c3), we see that for $q^{(2)}=3$ and $m^{(2)}=3$ a bandlike void appears between the dust shells when $A=3$. This is because the larger the charge and mass of the species 2 are, the lower is the drag force needed for forming the bandlike voids. Thus, at lower $A(=3)$ and larger charge $q^{(2)}(=3)$ and mass $m^{(2)}(=3)$, the different species can still be packed into different shells with a bandlike void between the shells. From Figs. 1(a4), 1(b4), and 1(c4), we can see that at a fixed $A=5$, the sizes of the band-like void and the entire dust-occupied region both increase rapidly with $q^{(2)}$ and $m^{(2)}$. Thus, the species charge and mass are independent factors in determining the system structure. For the same reason we can expect that in a single-species system only the center void can exist. Our

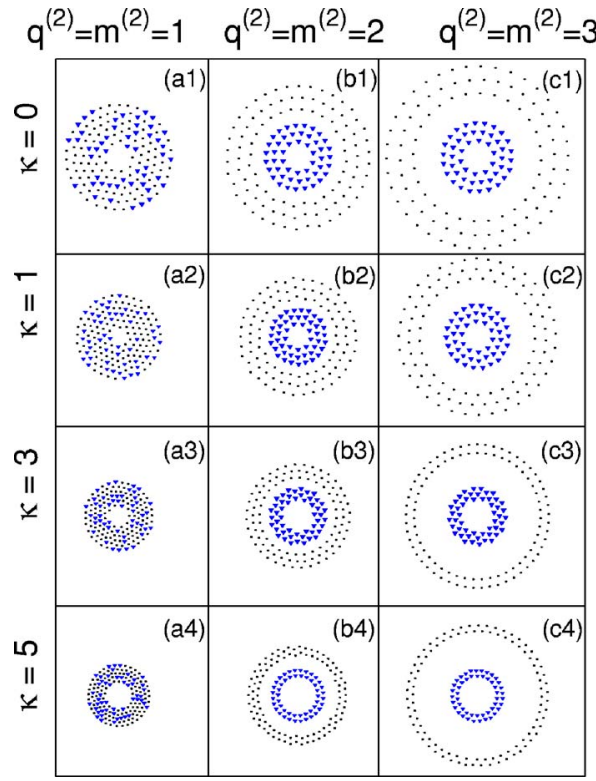

FIG. 2. (Color online) Minimum-energy configurations for two species of different charge and mass, but the same charge-to-mass ratio at different screening strength $\kappa$ at a fixed drag strength $A=5$. The total number of grains is $N=150$, where $N^{(1)}=50$ (triangles) and $N^{(2)}=100$ (dots). The subfigures are of the same arbitrary scale.

center- and band-void configurations are in good qualitative agreement with the cross-sectional dust structures observed in $3 \mathrm{D}$ dust systems $[15,16]$.

\section{Effect of screening}

We now investigate the effect of the screening strength $\kappa$. Figure 2 shows typical minimum-energy configurations of a two-species 150 dust grain system for $A=5$. Again, the special case of $q^{(2)}=q^{(1)}=1.0$ and $m^{(2)}=m^{(1)}=1.0$ is included as reference. In Fig. 2, the columns show the effect of the screening strength $\kappa$, and the rows show the effect of the charge $q^{(2)}$ and mass $m^{(2)}$. From the first column of Fig. 2, we see that when there is only one species, only a central void appears, and the size of the dust structure decreases with increase of $\kappa$, with the size of the central void almost unchanged. That is, the dust shell becomes more compact as $\kappa$ increases. This is because at a fixed $A$, increasing $\kappa$ would decrease the intergrain repulsive force, so that the inward confinement force pushes the grains closer to the center. The nearly constant size of the central void can be attributed to the fact that the outward drag force supporting it is unaffected by $\kappa$. When $\kappa=5$ [Fig. 2(a4)], the central void becomes somewhat larger than that at smaller $\kappa$ [see Figs. 2(a1)-2(a3)] because of reduced intergrain repulsion. Its boundary also becomes more circular.

From the second and third columns of Fig. 2, one can see that for larger differences in the species charge and mass, the different species also organize themselves into two distinct shells, with that of the smaller (larger) charge and mass located nearer to (farther away from) the center of the dust structure because of the different drag forces they experience. The dependence on the parameters are basically the 
same as already discussed above, so that with increase of $q^{(2)}$ and $m^{(2)}$, the size of the dust structure also increases. On the other hand, the sizes of the central and band voids, as well as the dust shells, are determined by the balancing of the various forces acting on the dust grains.

As discussed, with increasing $\kappa$, the shells of the different species become more compact. In fact, when $\kappa$ is large enough (and the attractive coefficient $\alpha$ remains small), the grain-grain interaction will be like hard spheres [30]. In this case, the sizes of the bandlike and the central disklike voids can be estimated. From Eq. (1), we can see that the first and second terms together represent a displaced harmonic potential, such as the ring-confinement potential of Schweigert et al. [41] for each species. In the displaced potential, the minimum is not at the system center $r=0$, but at $r=r_{*}$, i.e., the potential supports a central void of radius $r_{*}$. For the multispecies system, from the derivative $\partial_{r}\left[\left(m^{(k)} r_{*}^{(k) 2}-A q^{(k) 2} r_{*}^{(k)}\right)\right]$ $=0$, we can obtain the critical radius $r_{*}^{(k)}=A\left(q^{(k) 2} / 2 m^{(k)}\right)$ for species $k$. When the screening strength $\kappa$ is large and the attractive coefficient $\alpha$ is small, the shells of different species will be very compact. The size of the central disklike void is mainly determined by the smallest radius among the radii $r_{*}^{(k)}(k=1, \ldots, n)$, and the size of the bandlike void will be determined by the difference between the radii $r_{*}^{(i)}$ and $r_{*}^{(j)}$ of the shells $i$ and $j$ adjacent to the bandlike void. Therefore, at a given $A, q^{(2)}$ and $m^{(2)}$, with increasing $\kappa$, the sizes of the central disk void and the bandlike void will approach values determined by the critical radii $r_{*}^{(k)}(k=1, \ldots, n)$. In fact, in a system with a small $\kappa$, we can still use the critical radii $r_{*}^{(k)}$ to predict the void-size variation. For example, in Figs. 1(c1)2(c4), at the given $q^{(2)}=3$ and $m^{(2)}=3$, the disk-void size determined by $r_{*}^{(k)}=A\left(q^{(1) 2} / 2 m^{(1)}\right)$ and the band-void size determined by the difference $r_{*}^{(2)}-r_{*}^{(1)}=A\left(q^{(2) 2} / 2 m^{(2)}\right.$ $\left.-q^{(1) 2} / 2 m^{(1)}\right)$ increase with $A$. From Figs. 2(a4), 2(b4), and 2(c4), we can see that for $A=5$ and $\kappa=5$, the size of the bandlike void determined by the difference $r_{*}^{(2)}-r_{*}^{(1)}$ increases with $q^{(2)}$ and $m^{(2)}$, but the disk-void size determined by the radius $r_{*}^{(1)}$ does not change with $q^{(2)}$ and $m^{(2)}$ in Figs. 2(b4) and 2(c4) (the estimate by the critical radius $r_{*}^{(1)}$ is not valid for the degenerate case of Fig. 2(a4), where the species are indistinguishable). Hence, in a multispecies system, we can use the critical radii $r_{*}^{(k)}$ to predict the variation of the disk- and band-void sizes with $A, q^{(2)}$, and $m^{(2)}$. However, when $\kappa$ is not large, the size of a void will be determined by a balance between the intergrain force, the confinement force, and the drag force on the grains. From the above results, one can conclude that in a two-species system, the screening strength $\kappa$ and the charge $q^{(2)}$ and mass $m^{(2)}$ are independently important in determining the system structure. At a given $A$, the larger the values of $\kappa, q^{(2)}$, and $m^{(2)}$, the easier it is to form the bandlike void.

\section{B. Three-species systems}

From the above results, one can conclude that if the external drag force $A$ and the screening strength $\kappa$ are large enough, a central void as well as a bandlike void will appear in a two-species system. In Huang et al.'s [15] and Huang's

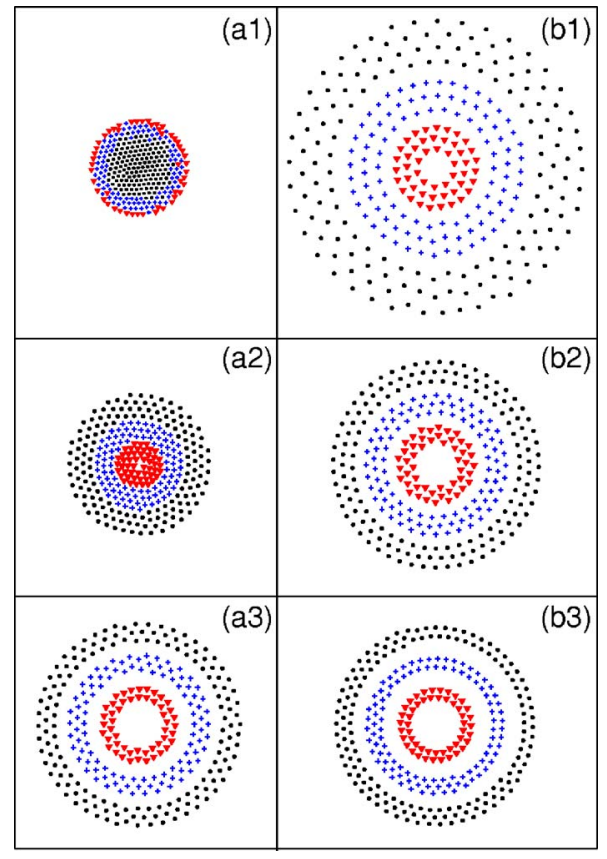

FIG. 3. (Color online) Minimum-energy configurations for a three-species system for $A=$ (a1) 0, (a2) 3, and (a3) 5, with fixed $\kappa=4$, and for $\kappa=$ (b1) 0 , (b2) 3 , and (b3) 5 , with fixed $A=5$. Here, $N=300, N^{(1)}=50$ (triangles), $N^{(2)}=100$ (crosses), $N^{(3)}=150$ (dots), $q^{(2)}=m^{(2)}=2$, and $q^{(3)}=m^{(3)}=3$. The subfigures are of the same arbitrary scale.

[16] experiments, multiple concentric band voids were also observed. These voids may have been caused by the presence of several species of grains, as can be expected during the evolution of the dust growth process $[15,16]$.

Accordingly, we consider the minimum-energy configurations of a larger system $(N=300)$ with three species of grains. Figure 3 shows the typical calculated minimumenergy configurations of a system containing three species of grains at different drag force strength $A$ with a fixed $\kappa=4$ [Figs. 3(a1)-3(a3)] and that at different screening strength $\kappa$ with a fixed drag force strength $A=5$ [Figs. 3(b1)-3(b3)]. The different grains again have different charge and mass, but have the same charge-to-mass ratio. From Figs. 3(a1)-3(a3), we can see that at a fixed $\kappa=4$, when $A=0$, the grains tend to separate, with those of the largest charge and mass located near to the system center, and the light ones located at the edge of the system, because the mass-dependent confinement force pushes the large grains closer to the system center to minimize the total system energy. With increasing $A$, the grains are separated into three shells, and the grains of the largest (smallest) charge and mass are located far away from (near to) the system center, due to the outward drag force on the different grains. When $A=5$, the central disk void and the concentric bandlike voids between the shells of the different grains occur. From Figs. 3(b1)-3(b3), we can see that at a fixed $A(=5)$, the different grains are separated into different shells. When $\kappa=0$, there are no clear bandlike voids formed between the shells. With increasing $\kappa$, the system size becomes smaller, and the shells become more compact. When $\kappa=5$, the concentric bandlike voids clearly appear between 


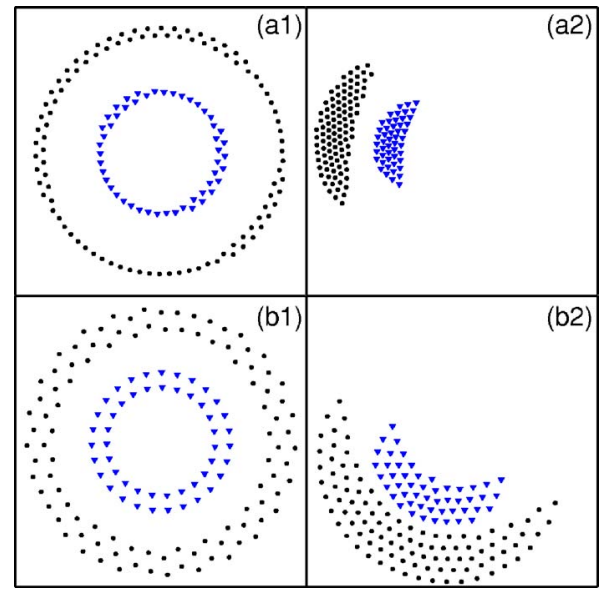

FIG. 4. (Color online) Asymmetrical minimum-energy states for a two-species system for (a1) $\kappa=15, \alpha=0$, (a2) $\kappa=15, \alpha=0.01$, (b1) $\kappa=5, \alpha=0$, and (b2) $\kappa=5, \alpha=0.04$, with fixed $A=5$. Here, $N=150$, $N^{(1)}=50 \quad$ (triangles), $N^{(2)}=100 \quad($ dots $)$, and $q^{(2)}=m^{(2)}=2$. The subfigures are of the same arbitrary scale.

the different shells. As discussed above, the reason is that with increasing $\kappa$, the intergrain repulsive force becomes lower, and the grains are pushed closer to the system center by the confinement force. At this case, the outward drag force and the inward confinement force the different grains experience lead to the bandlike void formation in the system. We also can see that when $\kappa=5$, the outer two shells of different grains are not uniform in thickness, which is due to the intergrain attraction forces that make the grains tend to stick together. In fact, when the attraction coefficient is large enough, the grains of the same species will stick together as one cluster, and no complete circle shells can be formed. We have also investigated much larger systems with more grains and species. The results show that the grains of the same species tend to populate in a common shell, and the central disklike voids as well as the bandlike voids can appear in the system when the drag force strength and the screening strength are large. The maximum number of possible shells formed of the various grains is equal to the total species number in the system.

\section{Highly asymmetrical states}

In the dust systems studied, all the forces involved are either radial or central. Although for multispecies systems, the centers of mass and charge of the (random) initial grain distributions do not in general coincide, one would expect that the minimum energy states should be symmetrical, unless the number of grains is too small. However, here radially outward drag force and attractive intergrain force are also involved, we can expect the existence of asymmetrical minimum-energy states, or even states containing clumps of dust grains, if for example, the repulsive intergrain force is too weak ( $\kappa$ large). In fact, Fig. 2(b4) and Fig. 3(b3) represent such states, but in a rather weak form.

To see if highly asymmetrical states can exist, we consider cases with large $\kappa$ and $\alpha$. Figure 4 is for two species with $q^{(2)}=m^{(2)}=2, N^{(1)}=50$ and $N^{(2)}=100, A=5$, and Fig. 4(a1) $\kappa=15, \alpha=0$, Fig. 4(a2) $\kappa=15, \alpha=0.01$, Fig. 4(b1) $\kappa=5, \alpha=0$, and Fig. 4(b2) $\kappa=5, \alpha=0.04$. One can see that, as expected, the attractive intergrain force and the radially outward drag force play important roles in forming the highly asymmetrical states. However, the marginal values of $\kappa$ and $\alpha$ allowing the formation of asymmetrical states also depend on all the other dust parameters and external forces. Asymmetrical states have been observed in the earlier simulations with a small number of grains [39], as well as in experiments $[15,16]$. However, in a real plasma, the effective intergrain and external forces in the bulk plasma can modify each other, so that it can be difficult to distinguish the cause and effect relationship in the formation of the asymmetrical states. This is especially so for the drag and the intergrain forces, since the grain charge is strongly dependent on the local electric field. We also note that the asymmetrical dust clumps are in general more compact and crystal-like.

\section{CONCLUSION}

Two-dimensional systems of multispecies charged grains interacting through a screened Coulomb repulsive potential plus a long-range attractive component added to it in a quadratic trap is studied by means of MD simulations. The grains are also subjected to an external drag force to simulate the effect of the ions streaming to the system boundary. It is found that in a multispecies system consisting of grains with different charge and mass, but having the same charge-tomass ratio, at a large screening strength without an external drag force, the grains with the large (small) charge and mass tend to be located near to (far away from) the system center, due to the inward mass-dependent confinement force on the grains. When the external drag force exists in the system, the different grains are separated into different shells, with those of the large (small) charge and mass located far away from (near to) the system center because of the different drag force the grains experience. When the screening strength is large, with increasing the drag force strength, a central void as well as the concentric bandlike voids between the different shells can appear. The void size increases with the drag force strength. At a given drag force strength, with increasing screening strength, the system size becomes smaller and the grain shells also become more compact. When the screening strength is large enough (with the intergrain attraction remaining small), the size of the central disklike void can be estimated by the smallest grain critical radius at which the grains have the minimal external potential energy, and the size of the bandlike void can be estimated by the difference of the critical radii of the different shells adjacent to the band void. The maximum number of possible shells formed of the different grains equals the total species number in the system.

Qualitatively, the void structures obtained in our simulation are consistent with that found in the horizontal slices of the 3D structures obtained in the dusty plasma experiments $[15,16]$. In the latter, the discharge is not symmetric in the vertical direction and the voids appear as domes and/or nested dome-shaped shells with their bottoms adjacent to the bottom electrode in the dust system. However, the results 
should be qualitatively applicable to the horizontal cross sections since the dynamic horizontal force balance process remains the same as in the $2 \mathrm{D}$ case. The occurrence of symmetrical and asymmetrical bandlike voids should be rather common in 2D dusty plasmas containing more than one species of dust grains. Our results may be useful as a guide to future experiments specifically designed for investigating the charge, mass, and size effects on the system structure and void formation. When specific configurations of the multispecies system can be observed experimentally, they can be used for diagnostics of the grain screening strength, attrac- tive intergrain force, and the ion drag force on the grains. It may also be useful for monitoring dust grain growth and obtaining information on a dusty plasma system by adding to it new species of dust grains.

\section{ACKNOWLEDGMENTS}

Y.H.L. acknowledges the National Science Foundation of China (Project No. 10205025) for initializing this work. Y.H.L. and Z.Y.C. would also like to thank a Bilateral Project between Flanders and China for financial support.
[1] A. Melzer, V. A. Schweigert, I. V. Schweigert, A. Homann, S. Peters, and A. Piel, Phys. Rev. E 54, R46 (1996).

[2] W. T. Juan, Z. H. Huang, J. W. Hsu, Y. J. Lai, and Lin I, Phys. Rev. E 58, R6947 (1998).

[3] M. H. Kong, W. P. Ferreira, B. Partoens, and F. M. Peeters, IEEE Trans. Plasma Sci. 32, 569 (2004).

[4] B. Liu, K. Avinash, and J. Goree, Phys. Rev. E 69, 036410 (2004).

[5] R. Vrancken, G. V. Paeva, G. M. W. Kroesen, and W. W. Stoffels, Plasma Sources Sci. Technol. 14, 509 (2005).

[6] J. B. Pieper, J. Goree, and R. A. Quinn, Phys. Rev. E 54, 5636 (1996).

[7] V. A. Schweigert, I. V. Schweigert, A. Melzer, A. Homann, and A. Piel, Phys. Rev. E 54, 4155 (1996).

[8] G. Praburam and J. Goree, Phys. Plasmas 3, 1212 (1996).

[9] V. N. Tsytovich, S. V. Vladimirov, and G. E. Morfill, Phys. Rev. E 70, 066408 (2004).

[10] V. N. Tsytovich, S. V. Vladimirov, G. E. Morfill, and J. Goree, Phys. Rev. E 63, 056609 (2001).

[11] D. Samsonov and J. Goree, Phys. Rev. E 59, 1047 (1999).

[12] J. Goree, G. E. Morfill, V. N. Tsytovich, and S. V. Vladimirov, Phys. Rev. E 59, 7055 (1999).

[13] M. R. Akdim and W. J. Goedheer, Phys. Rev. E 65, 015401(R) (2001).

[14] G. E. Morfill, H. M. Thomas, U. Konopka, H. Rothermel, M. Zuzic, A. Ivlev, and J. Goree, Phys. Rev. Lett. 83, 1598 (1999).

[15] F. Huang, M. F. Ye, L. Wang, and N. Jiang, Chin. Phys. Lett. 21, 121 (2004).

[16] F. Huang, D. Sc. dissertation, Institute of Physics, Beijing, 2005 (in Chinese).

[17] R. P. Dahiya, G. V. Paeva, W. W. Stoffels, E. Stoffels, G. M. W. Kroesen, K. Avinash, and A. Bhattacharjee, Phys. Rev. Lett. 89, 125001 (2002).

[18] N. D’Angelo, Phys. Plasmas 5, 3155 (1998).

[19] A. V. Ivlev, D. Samsonov, J. Goree, G. Morfill, and V. E. Fortov, Phys. Plasmas 6, 741 (1999).

[20] K. Avinash, Phys. Plasmas 8, 351 (2001).

[21] X. Wang, A. Bhattacharjee, S. K. Gou, and J. Goree, Phys.
Plasmas 8, 5018 (2001).

[22] I. Denysenko, M. Y. Yu, L. Stenflo, and N. A. Azarenkov, Phys. Plasmas 12, 042102 (2005).

[23] M. Kretschmer, S. A. Khrapak, S. K. Zhdanov, H. M. Thomas, G. E. Morfill, V. E. Fortov, A. M. Lipaev, V. I. Molotkov, A. I. Ivanov, and M. V. Turin, Phys. Rev. E 71, 056401 (2005).

[24] Y. H. Liu, Z. Y. Chen, F. Huang, M. Y. Yu, L. Wang, and A. Bogaerts, Phys. Plasmas 13, 052110 (2006).

[25] Z. Y. Chen, M. H. Kong, M. V. Milošević, and Y. C. Wu, Phys. Scr. 67, 439 (2003).

[26] V. N. Tsytovich, N. G. Gousein-zade, and G. E. Morfill, Phys. Plasmas 13, 033503 (2006).

[27] Z. Y. Chen, M. Y. Yu, and H. Q. Luo, Phys. Scr. 64, 476 (2001).

[28] A. G. Bashkirov, Phys. Rev. E 69, 046410 (2004).

[29] V. N. Tsytovich and G. E. Morfill, Plasma Phys. Rep. 28, 171 (2002).

[30] B. Liu and J. Goree, Phys. Rev. Lett. 94, 185002 (2005).

[31] V. N. Tsytovich, Y. K. Khodatev, and R. Bingham, Comments Plasma Phys. Controlled Fusion 17, 249 (1996).

[32] Y. P. Chen, H. Q. Luo, M. F. Ye, and M. Y. Yu, Phys. Plasmas 6, 699 (1999).

[33] L. Wang, Comments Plasma Phys. Controlled Fusion 1, 117 (1999).

[34] J. Allen and B. Annoratone, J. Plasma Phys. 7, 397 (1999).

[35] E. Tomme, E. B. Annaratone, and J. Allen, Plasma Sources Sci. Technol. 9, 87 (2000).

[36] V. M. Bedanov and F. M. Peeters, Phys. Rev. B 49, 2667 (1994).

[37] J. A. Drocco, C. J. Olson Reichhardt, C. Reichhardt, and B. Jankó, Phys. Rev. E 68, 060401(R) (2003).

[38] K. Nelissen, B. Partoens, and F. M. Peeters, Phys. Rev. E 69, 046605 (2004).

[39] W. P. Ferreira, F. F. Munarin, K. Nelissen, R. N. Costa Filho, F. M. Peeters, and G. A. Farias, Phys. Rev. E 72, 021406 (2004).

[40] Y. H. Liu, Z. Y. Chen, M. Y. Yu, L. Wang, and A. Bogaerts, Phys. Rev. E 73, 047402 (2006).

[41] I. V. Schweigert, V. A. Schweigert, and F. M. Peeters, Phys. Rev. B 54, 10827 (1996). 